\title{
Comparative Analysis of Volumetric High-Resolution Heavily T2-Weighted MRI and Time-Resolved Contrast-Enhanced MRA in the Evaluation of Spinal Vascular Malformations
}

\author{
(D) S.K. Kannath, (DS. Mandapalu, DB. Thomas, (D). Enakshy Rajan, and (D). Kesavadas
}

\begin{abstract}
BACKGROUND AND PURPOSE: Volumetric high-resolution heavily T2-weighted imaging or time-resolved contrast-enhanced MRA is used in the detection and characterization of spinal vascular malformations, though inherent trade-offs can affect their overall sensitivity and accuracy. We compared the efficacy of volumetric high-resolution heavily T2-weighted and time-resolved contrast-enhanced images in spinal vascular malformation diagnosis and feeder characterization and assessed whether a combined evaluation improved the overall accuracy of diagnosis.
\end{abstract}

MATERIALS AND METHODS: Twenty-eight patients with spinal vascular malformations (spinal dural arteriovenous fistula, spinal cord arteriovenous malformation, and perimedullary arteriovenous fistula) were prospectively enrolled. MR images were separately analyzed by 2 neuroradiologists blinded to the final diagnosis.

RESULTS: Both sequences demonstrated 100\% sensitivity and $93.5 \%$ accuracy for the detection of spinal vascular malformations. Volumetric high-resolution heavily T2-weighted imaging was superior to time-resolved contrast-enhanced MR imaging for identification of spinal cord arteriovenous malformations (100\% versus $90 \%$ sensitivity and $96.7 \%$ versus $93.5 \%$ accuracy), however, for the diagnosis of perimedullary arteriovenous fistula, time resolved contrast enhanced MRI was found to perform better than the volumetric T2 sequence ( $80 \%$ versus $60 \%$ sensitivity and $96.7 \%$ versus $93.5 \%$ accuracy). Both sequences showed equal sensitivity ( $100 \%$ ) and accuracy ( $87 \%$ ) for spinal dural arteriovenous fistulas. Combined evaluation improved the overall accuracy across all types of spinal vascular malformation. Volumetric high-resolution heavily T2-weighted imaging was superior or equal to time-resolved contrast-enhanced MR imaging for feeder identification of spinal dural arteriovenous fistulas for both observers ( $90.9 \%$ and $72.7 \%$ versus $72.7 \%$ ), which improved to $90.9 \%$ when the sequences were combined. Time-resolved contrast-enhanced MR imaging performed better for major and total feeder identification of spinal cord arteriovenous malformation ( $80 \%$ versus $60 \%$ ) and perimedullary arteriovenous fistula ( $80 \%$ versus $60 \%-80 \%$ ).

CONCLUSIONS: Combined volumetric high-resolution heavily T2-weighted imaging and time-resolved contrast-enhanced MR imaging can improve the sensitivity and accuracy of spinal vascular malformation diagnosis, classification, and feeder characterization.

ABBREVIATIONS: ASA = anterior spinal artery; $\mathrm{PMAVF}=$ perimedullary arteriovenous fistula; $\mathrm{SCAVM}=$ spinal cord arteriovenous malformation; $\mathrm{SDAVF}=$ spinal dural arteriovenous fistula; SVM = spinal vascular malformation; TRCE = time-resolved contrast-enhanced; TRICKS = time-resolved imaging of contrast kinetics; $\mathrm{VHHT2WI}=$ volumetric high-resolution heavily T2-weighted imaging

S pinal vascular malformations (SVMs) are rare entities at various locations in the spinal cord and its coverings. These include predominantly spinal cord arteriovenous malformation (SCAVM), spinal dural arteriovenous fistula (SDAVF), and perimedullary arteriovenous fistula (PMAVF). The clinical presentation is variable, though typical clinical patterns exist among

Received May 9, 2019; accepted after revision July 1.

From the Department of Imaging Sciences and Interventional Radiology (S.K.K., B.T., J.E.R., C.K.), Sree Chitra Tirunal Institute for Medical Sciences and Technology, Trivandrum, Kerala, India; and Department of Radiology (S.M.), NRI Medical College, Chinakakani, Guntur, Andra Pradesh, India.

Please address correspondence to Santhosh Kumar Kannath, MD, PDCC, Department of Imaging Sciences and Interventional Radiology, SCTIMST, Medical College PO, Trivandrum, 695011 Kerala, India; e-mail: drsanthoshkannath@gmail.com certain entities such as acute spinal cord dysfunction due to hemorrhage in SCAVM or slow progressive paraplegia in SDAVFs. ${ }^{1,2}$ There is a variable delay in the diagnosis due to nonspecific clinical symptoms or low clinical suspicion, which often leads to serious morbidity, even after successful treatment. MR imaging is usually the initial investigation of choice based on which diagnosis is suspected, while DSA remains the criterion standard. ${ }^{3}$ DSA is, however, invasive and has additional risks such as exposure to ionizing radiation, iodinated contrast medium overload, and a

Indicates article with supplemental online tables.

Indicates article with supplemental on-line photo.

http://dx.doi.org/10.3174/ajnr.A6164

AJNR Am J Neuroradiol 40:1601-06 Sep 2019 www.ajnr.org 
small risk of inducing spinal cord infarction. ${ }^{4}$ DSA also can miss the diagnosis in SDAVF and PMAVF because of operator-dependent factors, and such patients may have a poor prognosis even after successful treatment. ${ }^{5,6}$

SVMs are suspected on the basis of the presence of flow voids in the subarachnoid space and/or cord parenchyma and are further evaluated using volumetric high-resolution heavily T2-weighted imaging (VHHT2WI), contrast-enhanced MRA, and time-resolved contrast-enhanced (TRCE) MR imaging sequences for characterization and localization of arterial feeders. ${ }^{7-13}$ VHHT2WI sequences such as Cube (GE Healthcare, Milwaukee, Wisconsin) and sampling perfection with application optimized contrasts by using different flip-angle evolution (SPACE; Siemens, Erlangen, Germany) provide excellent contrast between the cord and CSF along with suppression of CSF flow artifacts. Submillimeter spatial resolution of these sequences allows multiplane reconstruction without artifacts, which would help in the detection and diagnosis of SVM and localization of arterial feeders. ${ }^{13}$ On the contrary, dynamic TRCE MR image sequences such as time-resolved imaging of contrast kinetics (TRICKS) provide good temporal resolution and capture contrast kinetics as it transits through the feeding artery and vascular malformation into the draining vein. While the lack temporal information is a major disadvantage for the former sequence, the latter sequence has poor spatial resolution and a limited FOV; thus, an accurate localization or characterization of an SVM may be difficult or lesions may be missed altogether. ${ }^{10}$ We hypothesized that combining these 2 sequences could maximize the overall diagnostic performance while offsetting the trade-offs and thus could potentially improve the overall accuracy of detection and characterization of spinal vascular malformations. In the present study, we used the diagnostic capability and feeder characterization of VHHT2WI (Cube) and TRCE MR (TRICKS) independently and in combination for the assessment of SVM.

\section{MATERIALS AND METHODS}

This prospective observational study included all patients with clinically suspected or diagnosed spinal vascular malformations who presented to the radiology department of Sree Chitra Tirunal Institute for Medical Sciences and Technology between May 2015 and October 2017. Patients with contraindications to MR imaging or partially treated SVMs or those who were unwilling to give consent were excluded from the study. MR imaging of the whole spine was performed on a 3T Discovery $750 \mathrm{MR}$ imaging scanner (GE Healthcare) initially, followed by invasive spinal angiography on an Innova 3131 biplane DSA machine (GE Healthcare).

\section{MR Imaging Technique}

The MR imaging protocol consisted of initial routine T2 and 3D Cube acquisitions of the whole spine in the sagittal plane, followed by contrast-enhanced TRICKS of the suspected region. The parameters were as follows-Cube: TE/TR, 60-115/2500 ms; echo-train length, 90 ; FOV, $30 \mathrm{~cm}$ with a matrix of $288 \times 288$ and slice thickness of $1.6 \mathrm{~mm}$ in the sagittal plane. The entire spine was covered in 2 or 3 stations. The total acquisition time for each station was 6.08 minutes. For TRICKS, because there is a limita- tion to the longitudinal area of coverage, the FOV was centered at the region of maximum concentration of flow voids, after evaluating the prior conventional T2 sequence. Initially the TRICKS sequence with a temporal resolution of 4 seconds and 200 milliseconds was used for the first 7 patients, and the parameters were as follows: TE/TR 1.4-11/4 ms; flip angle, $30^{\circ}$; slice thickness, 2 $\mathrm{mm}$ with an FOV of $46 \mathrm{~cm}$, matrix of $512 \times 256$, and NEX of 1 with 20 phases acquired in the sagittal plane in 1 minute and 43 seconds. To shorten the temporal resolution to 3 seconds 100 milliseconds, we reduced the TR to $3.7 \mathrm{~ms}$, the NEX to 0.75 , and the flip angle to $20^{\circ}$, resulting in a total scan time of 1 minute 15 seconds, and these parameters were used in the remainder of the study. Gadolinium contrast medium (gadodiamide, Omniscan; GE Healthcare, Piscataway, New Jersey), $0.2 \mathrm{mmol} / \mathrm{kg}$ body weight $(15-18 \mathrm{~mL})$, was injected at a rate of $3.5 \mathrm{~mL} / \mathrm{s}$ followed by a 20 -mL saline chase follow-through.

The resulting images from both sequences were postprocessed using multiplanar reconstructions and maximum intensity projections in orthogonal and oblique planes on an Advantage Workstation for Windows (GE Healthcare).

\section{Data Analysis}

The VHHT2WI and TRCE MR images were independently analyzed after a gap of 2 weeks for the presence of vascular malformations, type (SDAVF, SCAVM, or PMAVF), and feeding arteries (number and their level) by 2 neuroradiologists (S.K.K. with 9 years' experience and S.M. with 3 years' experience) who were blinded to the case particulars. The spinal angiography data were analyzed by a single neuroradiologist (J.E.R. with 14 years' experience) blinded to the MR imaging observations. The technique of identification of the type of vascular malformation and its feeder was discussed previously and is detailed in On-line Table $2 .^{10,13}$ Statistical analysis of the data was performed by calculating the sensitivity, specificity, accuracy, and positive and negative predictive values of VHHT2WI, TRCE, and combined data in identifying the type and location of vascular malformations in comparison with spinal angiography. $\kappa$ coefficients of intermodality and interobserver variability were also calculated. All analyses were performed on SPSS, Version 21 (IBM, Armonk, New York).

\section{RESULTS}

Of 34 patients who met the inclusion criteria, 28 patients were included in the final analysis after exclusions (On-line Figure). The median age of the patients was 48 years (range, 15-78 years), with 22 males and 6 females. Eleven patients had SDAVFs, 10 had SCAVMs, 5 patients had PMAVFs, and the remaining 2 patients had extraspinal arteriovenous malformations. The clinical and demographic characteristics of the subjects are shown in Table 1.

\section{Diagnosis and Characterization of SVMs}

Both VHHT2WI and TRCE MR imaging performed equally with a sensitivity of $100 \%$ and accuracy of $93.5 \%$ for diagnosing the presence of SVMs. The specificity was low (33\%), due to a low number of true-negative cases.

For the diagnosis of SDAVF, VHHT2WI and TRCE MR imaging had equal sensitivity, specificity, and accuracy of $100 \%$, 
$80 \%$, and $87 \%$, respectively. The VHHT2WI sequence misdiagnosed 2 cases of PMAVFs and 1 case of vertebral body AVM as DAVFs. The false diagnosis of the SDAVF was made due to the presence of flow voids without apparent identification of a nidus. False-positives occurred in 3 cases with TRCE MR imaging: In 1 case of an SCAVM, a small nidus was misinterpreted as a draining vein; in another case of PMAVF, the anterior spinal artery (ASA) was not visualized and was misdiagnosed as a DAVF; and in the last, in an extraspinal AVM, the nidus was missed. After we combined both sequences, the specificity and accuracy improved to $84 \%$ and $90 \%$, respectively. Thus, cross-confirmation between the 2 modalities improved the overall accuracy of diagnosis.

For SCAVM, the VHHT2WI had excellent sensitivity and accuracy of $100 \%$ and $96.7 \%$, respectively, while the TRCE MR imaging had a sensitivity and accuracy of $90 \%$ and $93.5 \%$, respectively. Both sequences had an equal specificity of $95.2 \%$. One patient with an extraspinal AVM and one with a spinal cord AVM were misdiagnosed with DAVFs on TRCE imaging. Combining the sequences improved the overall accuracy of TRCE imaging to that of VHHT2WI.

For PMAVFs, TRCE MR imaging performed better, with a sensitivity and accuracy of $80 \%$ and $96.7 \%$ compared with the VHHT2WI sequence, which had a lower sensitivity and accuracy of $60 \%$, and $93.5 \%$, respectively. Both sequences had a specificity and positive predictive value of $100 \%$. In 2 patients, the PMAVF

\begin{tabular}{|c|c|c|c|}
\hline & SDAVF & SCAVM & PMAVF \\
\hline Median age (range) (yr) & $57.7(49-67)$ & $29(15-53)$ & $52(27-78)$ \\
\hline \multicolumn{4}{|l|}{ Sex } \\
\hline Male & 10 & 6 & 5 \\
\hline Female & 1 & 4 & 0 \\
\hline \multicolumn{4}{|l|}{ Comorbidities } \\
\hline Diabetes & 4 & 1 & 2 \\
\hline Hypertension & 2 & 0 & 1 \\
\hline \multicolumn{4}{|l|}{ Presentation } \\
\hline Acute/subacute & 0 & 8 & 1 \\
\hline Chronic & 11 & 2 & 4 \\
\hline \multicolumn{4}{|l|}{ Clinical features } \\
\hline \multicolumn{4}{|l|}{ Initial symptoms } \\
\hline Low back pain & 3 & 7 & 3 \\
\hline Sensory symptoms & 3 & 5 & 2 \\
\hline Motor weakness & 5 & 5 & 0 \\
\hline \multicolumn{4}{|l|}{ Symptoms at diagnosis } \\
\hline All (motor/sensory/bladder/bowel) & 9 & 7 & 5 \\
\hline Any symptoms & 2 & 3 & 0 \\
\hline Time to diagnosis (median) (range) (mo) & $11.4(4-24)$ & $42.6(0-180)$ & $13.4(1-24)$ \\
\hline
\end{tabular}

was falsely diagnosed as an SDAVF on VHHT2WI. The combined evaluation improved the sensitivity to that of TRCE MR imaging due to better identification of the ASA.

\section{Feeder Identification of SVMs}

DAVF. The level of the arterial feeder to a DAVF was exactly identified by the VHHT2WI sequence in 7 and 6 of 11 patients by observers $1(63.6 \%)$ and $2(54.5 \%)$, respectively. When extended to 1 vertebral level, observer 1 identified arterial feeders in 10 of 11 (90.9\%) patients, whereas observer 2 identified them in 8 of 11 patients $(72.7 \%)$. On the TRCE sequence, observers 1 and 2 identified the level in 8 of $11(72.7 \%)$ patients. By combined evaluation, both observers identified feeders in 10 of 11 patients (Table 2 ). The laterality of the arterial feeder was correctly predicted in all cases by observer 1 but was misinterpreted in 1 case by observer 2 . In the presence of multiple feeders to the DAVF, only 1 feeder could be identified by both observers.

AVM. Both the observers identified dominant arterial feeders in 6 of 10 patients on VHHT2WI and 8 of 10 patients on the TRCE sequence (Table 2). On the TRCE sequence, 2 feeders (20\%) were falsely localized to an incorrect level by both observers.

Also, TRCE performed better in the assessment of the overall number of feeders by both observers compared with VHHT2WI. More than half the number of feeders were identified by TRCE $(54.0 \%-66.6 \%)$, while identification was possible in only $25 \%-29 \%$ with VHHT2WI. Intranidal aneurysms were found on spinal angiography in 4 patients. Both observers were able to identify these in 3 of 4 patients (75\%) using both sequences.

PMAVF. The dominant feeder or single feeder could be identified on VHHT2WI in 4 of $5(80 \%)$ patients by observer 1 , and in 3 of $5(60 \%)$ patients by observer 2. Similarly, both observers could identify feeders in 4 of $5(80 \%)$ patients with the TRCE sequence.

Of 7 feeders on spinal angiography, on the VHHT2WI sequence, observers 1 and 2 identified 4 (57.1\%) and $3(42.8 \%)$ arterial feeders, respectively. Using the TRCE sequence and combined VHHT2WI and TRCE MR images, observers 1 and 2 iden-

Table 2: Feeder characterization and level localization of various spinal vascular malformations using different MR imaging sequences

\begin{tabular}{|c|c|c|c|c|c|c|}
\hline & \multicolumn{2}{|c|}{ VHHT2WI } & \multicolumn{2}{|c|}{ TRCE MRA } & \multicolumn{2}{|c|}{ Combined Approach } \\
\hline & Obs. 1 & Obs. 2 & Obs. 1 & Obs. 2 & Obs. 1 & Obs. 2 \\
\hline \multicolumn{7}{|l|}{ SDAVF } \\
\hline Exact level & $7 / 11(64 \%)$ & $6 / 11(54 \%)$ & $7 / 11(64 \%)$ & $7 / 11(64 \%)$ & $7 / 11(64 \%)$ & $7 / 11(64 \%)$ \\
\hline Within 1 vertebral level & 10/11 (91\%) & 8/11 (73\%) & 8/11 (73\%) & $8 / 11(73 \%)$ & 10/11 (91\%) & 10/11 (91\%) \\
\hline \multicolumn{7}{|l|}{ SCAVM } \\
\hline Dominant feeder & $6 / 10(60 \%)$ & $6 / 10(60 \%)$ & $8 / 10(80 \%)$ & $8 / 10(80 \%)$ & $8 / 10(80 \%)$ & $8 / 10(80 \%)$ \\
\hline All feeders & $6 / 24(25 \%)$ & $7 / 24(29 \%)$ & $13 / 24(54 \%)$ & $16 / 24(67 \%)$ & $13 / 24(24 \%)$ & $16 / 24(67 \%)$ \\
\hline \multicolumn{7}{|l|}{ PMAVF } \\
\hline Dominant feeder & $4 / 5(80 \%)$ & $3 / 5(60 \%)$ & $4 / 5(80 \%)$ & $4 / 5(80 \%)$ & $4 / 5(80 \%)$ & $4 / 5(80 \%)$ \\
\hline All feeders & $4 / 7(57 \%)$ & $3 / 7(43 \%)$ & $4 / 7(57 \%)$ & $5 / 7(71 \%)$ & $4 / 7(57 \%)$ & $5 / 7(71 \%)$ \\
\hline
\end{tabular}

Note:-Obs. Indicates observer. 


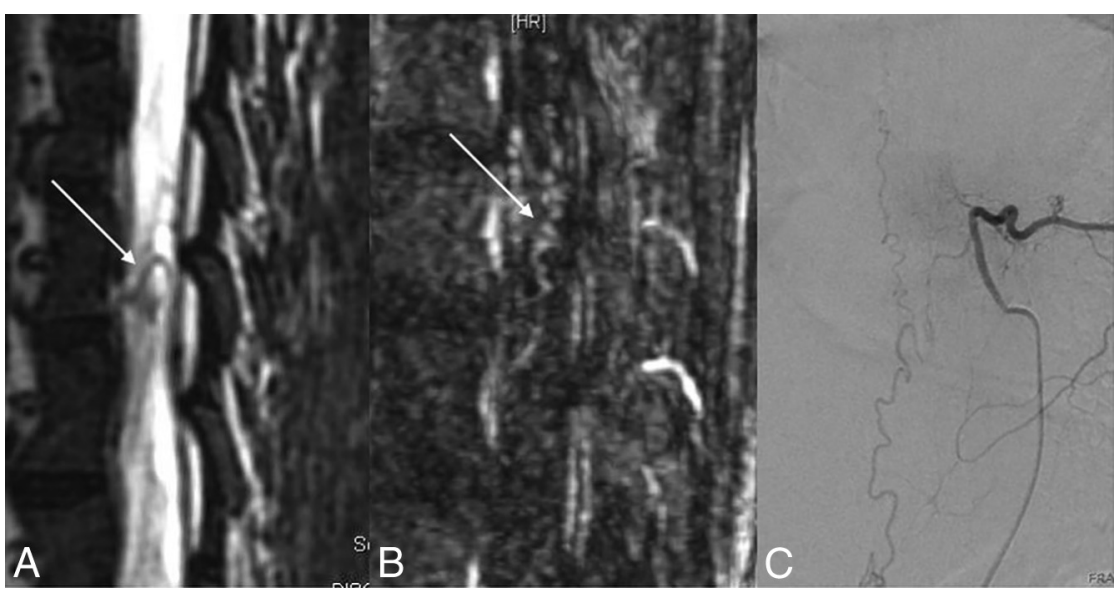

FIG 1. SDAVF. The VHHT2WI (A) shows a radicular vein (arrow) traced to left D8 vertebral foramen. The vein is faintly appreciated in TRCE (arrow in B) as well. The diagnosis is confirmed on DSA (C).

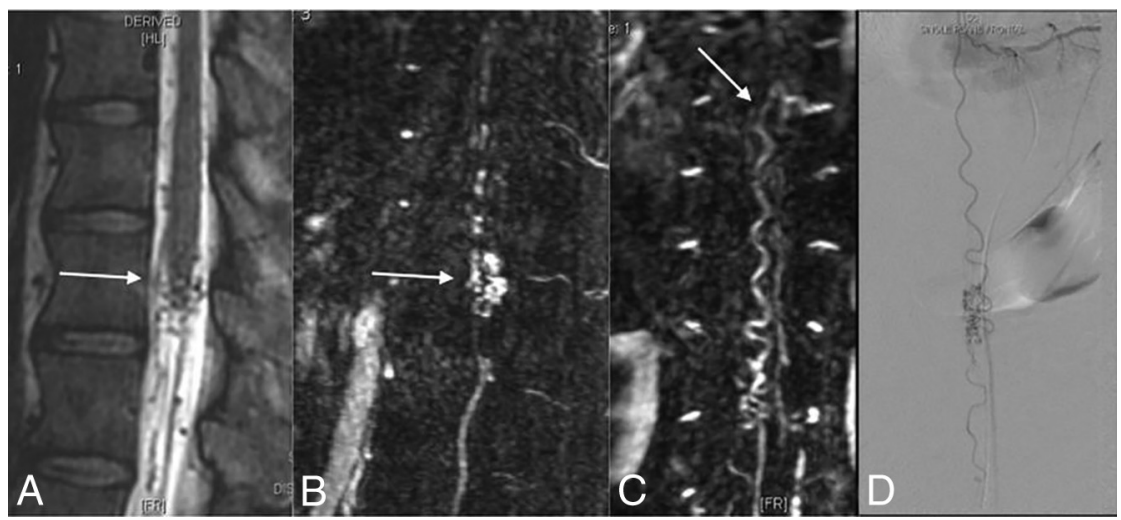

FIG 2. SCAVM. VHHT2WI (A) shows flow voids (arrow) within the conus suggesting the diagnosis of SDAVF. The feeder to the SCAVM was not identified. The parenchymal nidus (arrow in $B$ ) and the feeder from the ASA (arrow in C) are well seen in TRCE MR imaging. DSA confirms the observation $(D)$.

tified $4(57.1 \%)$ and $5(71.4 \%)$ feeders, respectively. On the TRCE sequence, 1 false-positive was identified by observer 1 and 2 falsepositives were identified by the observer 2 . Two of 5 patients had multiple feeders. VHHT2WI could identify only a single feeder level in 2 patients by both observers. However, by TRCE, observer 2 could identify both feeders in 1 patient, and observer 1 could identify only 1 feeder.

The representative cases of the 3 types of SVMs are illustrated in Figs 1-3.

\section{Intermodality and Interobserver Agreement}

Moderate-to-substantial agreement was noted between the 2 modalities (VHHT2WI and TRCE MR) among the 2 observers in the evaluation of the feeders or for localization. However, notably, the agreement improved for both the observers when a combined evaluation was performed. Substantial-to-perfect interobserver agreement was also noted across the modalities for all types of SVMs (On-line Tables 1 and 2).

\section{DISCUSSION}

Advanced MR imaging techniques used for the evaluation of SVMs include anatomic depiction of the vascular malformation using VHHT2WI (SPACE or Cube) or TRCE MR angiographic sequences (TRICKS). Most of the earlier studies discuss the utility of a specific MR image for a particular spinal malformation such as an SDAVF or SCAVM. To our knowledge, our series is the first study to report the usefulness of 2 different imaging sequences in the evaluation of spinal vascular malformation and its characterization. Our study found that VHHT2WI and TRCE MR imaging had an overall sensitivity of $100 \%$ and accuracy of $93.5 \%$ in the evaluation of suspected spinal vascular malformations. Also, the combined evaluation did not improve the accuracy of detection, suggesting that any one of the modalities is sufficient for confirming the presence of a vascular malformation. For feeder identification of an SDAVF, volumetric sequences were found to have higher accuracy, and combined evaluation yielded a detection rate within 1 vertebral level of $90 \%$. However, the major feeders of SCAVMs and PMAVFs were better recognized using the TRCE sequence than the volumetric sequence.

Conventional T2 MR imaging is often the first diagnostic study in the evaluation of spinal vascular malformations. The presence of flow voids and cord hyperintensity in a patient with a suspected spinal vascular malformation is reported to have $100 \%$ sensitivity in the diagnosis of SDAVFs. ${ }^{3}$ Flow voids, however, are observed in only about $70 \%$ of cases; moreover, the detection rate could be very low $(<40 \%)$ in low-flow shunt lesions. ${ }^{3,14}$ Advanced MR images can improve the sensitivity and diagnostic accuracy for the detection of SVM. Amarourche et $\mathrm{al}^{10}$ reported the utility of the TRCE MR imaging technique (TRICKS) in the evaluation of different types of SVMs. In this study, $98 \%$ sensitivity and $63 \%$ specificity were achieved for the diagnosis of SVMs. Although the characterization of SDAVFs was excellent with this technique, when one included the localization of feeders within 1 level (82.8\%), a false-positive interpretation was observed for PMAVFs, and the proportion of SCAVMs included in the study was very low $(6.4 \%)$. Other studies on SDAVFs also reported similar high sensitivity $(88 \%-100 \%)$ and specificity $(80 \%-90 \%)$ for the detection of SDAVFs and feeder localization $(85.7 \%-100 \%) .{ }^{11,12}$ The performance of TRCE is affected by several technical parameters, such as positioning of the FOV, delay between scan acquisitions and contrast bolus initiation, and temporal resolution of the sequence. ${ }^{10-12}$ Positioning of the FOV outside the region of the fistula can lead to a false-negative diagnosis, while low temporal resolution can lead to inadequate mapping of high- or low-volume shunts, yielding an erroneous interpretation. ${ }^{10-12}$

The other sequence used to investigate SVMs is VHHT2WI, 


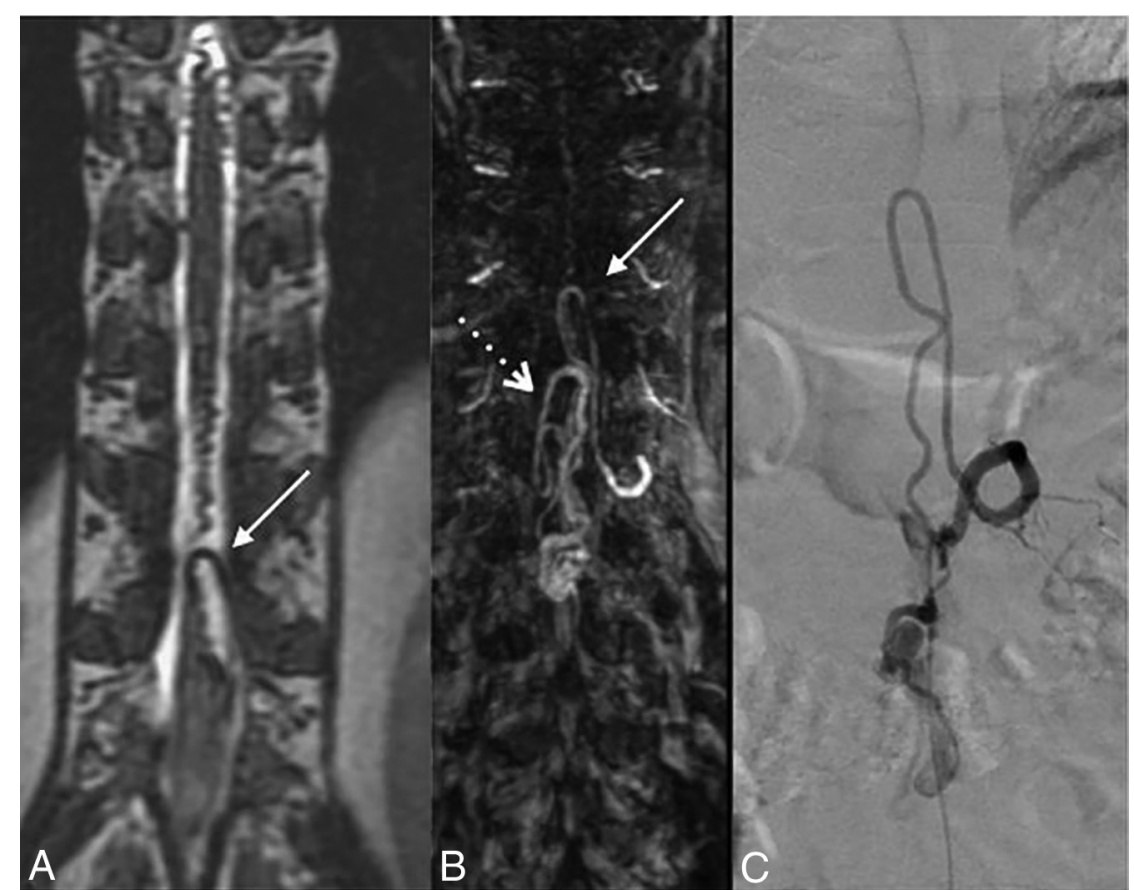

FIG 3. PMAVF. VHHT2WI shows a prominent ASA (arrow in A) supplying the fistula (not shown). TRCE MR image shows 2 ASAs feeding the fistula (arrows in B). A hypertrophied ASA supplying the fistula is demonstrated in $C$.

which provides superior parenchymal-CSF contrast distinction, thus allowing easy identification of even subtle flow voids and further characterization of the malformations based on the localization of the lesion or the feeder that supplies it. ${ }^{13,15} \mathrm{~A}$ recent study using this sequence demonstrated $98.1 \%$ sensitivity and $90 \%$ specificity for the diagnosis of SVMs, which was higher for SDAVFs (100\% and 90\%) compared with SCAVMs ( $89 \%$ and $71.8 \%$ ), respectively. ${ }^{16}$ Additionally, VHHT2WI could be used for localization of SDAVFs, and the site could be reliably identified within 1 vertebral level in $94 \%$ of the cases. ${ }^{13}$ However, the role of this sequence to distinguish intradural vascular malformations such as PMAVFs and SCAVMs and the capability of identifying feeders to these malformations are unknown. Thus, although both VHHT2WI and TRCE MR imaging are excellent tools to evaluate suspected SVMs, due to inherent technical limitations of each of these sequences, whether it is possible to replace a diagnostic spinal angiography or plan limited angiography with therapeutic intent is unclear. ${ }^{10,11,17}$

In our study, we found that VHHT2WI and TRCE MR imaging had high sensitivity and accuracy in the diagnosis of SVM, concurrent with other reported studies in the literature. ${ }^{10-15}$ Although the diagnosis of SDAVF could be confidently made using either sequence, a false-positive diagnosis was a concern for both. In our series, 2 cases of PMAVF and 1 case each of PMAVF and SCAVM were erroneously diagnosed as SDAVFs. Although VHHT2WI has submillimeter resolution, a near-normal-sized ASA feeding a low-flow PMAVF (type A PMAVF) might be missed due to volume averaging and thus could be erroneously reported as an SDAVF. Similarly, due to the low temporal resolution of TRCE MR imaging, optimal capture of different angiographic phases might be a limitation in a slow-flow PMAVF or a small
SCAVM with a near-normal-sized ASA feeder, resulting in an overlap of feeders and draining veins. ${ }^{10}$ Combining both sequences led to a higher accuracy of $90 \%$, and this improvement is likely a consequence of improved diagnostic confidence of subtle imaging observations in one technique, which could be crossconfirmed with the other. The higher sensitivity and accuracy of VHHT2WI and TRCE MR imaging in the diagnosis of SCAVMs in our series are not surprising, given both the sequences' ability in direct visualization of the parenchymal nidus, which, in itself, is confirmatory. However, TRCE MR imaging was found to be superior in the depiction of PMAVFs, primarily due to its ability to demonstrate the arterial feeder.

Similar to results of prior reports, our results confirm the role of advanced MR images such as VHHT2WI and TRCE in the detection of arterial feeders to SDAVFs. The detection rate for both sequences within 1 vertebral level was found to be high and comparable with those reported in the literature. ${ }^{10-13}$ Most interesting, we observed that the rate of identification depended on the readers' experience; however, by using input from both the sequences, high accuracy $(90.9 \%)$ could be attained. This suggests that the effect of the learning curve on interpretation could be offset by using 2 modalities rather than a single sequence. A similar observation was also noted for PMAVF, in which a combined evaluation detected a higher proportion of the main as well as all feeders. Contrary to the prior report, both sequences could identify main feeders in $60 \%-80 \%$ of the cases and, in more than half, the number of all feeders to SCAVMs. ${ }^{10}$ The poor characterization reported by Amarouche et $\mathrm{al}^{10}$ might be due to limited experience; only 3 patients with SCAVMs were included in their study cohort. High interrater agreement was found for all the parameters in our study, suggesting that the observations are largely unambiguous and reproducible.

In this study, we used 2 disparate MR imaging techniques to evaluate anatomic and flow characteristics of SVMs. Although the performances of these individual sequences are comparable as far as diagnosis and feeder identification of various SVMs is concerned, combined evaluation improved the overall accuracy. The greatest utility of the combined sequence was found in enhancing the specificity and accuracy of SDAVF by discriminating it from PMAVF and identification of the dominant feeders to intradural malformations such as PMAVF and SCAVM. One disadvantage of using both sequences for SVM evaluation is the mild prolongation of the total acquisition time. However, the clinician could opt for any particular sequence for the initial evaluation, and additional sequences could be used only if the findings are confirmatory of the pres- 
ence of an SVM. The current favored approach at the authors' institution is to obtain VHHT2WI initially, and perform a TRCE sequence later, centering the FOV at the site of the SVM localized from the earlier sequence. With this approach, falsenegative interpretation due to improper placement of the FOV in TRICKS could be avoided and the observations could be confirmed by both modalities. Accurate distinction of spinal vascular malformations and their feeders preoperatively can aid the interventionist in tailoring the spinal DSA and planning endovascular treatment in a single sitting. This approach will have the advantage of reduction in procedural time and the risks of spinal DSA, radiation exposure, and contrast load along with an overall reduced hospital stay. ${ }^{17}$

Our study has several strengths. The study design was prospective, blinded, and performed on a 3T MR imaging machine. Spinal vascular malformations were evaluated with 2 different sequences, which enabled characterization of both the anatomic and physiologic aspects of the pathology. Furthermore, we were able to overcome the relative drawback of the limited FOV with the TRCE sequence by planning the FOV on the basis of findings on conventional MR imaging rather than using a fixed position as in prior studies. Also, different types of SVMs were included and separately analyzed to understand the disease-specific performance of the sequences.

There are a few limitations to our study. Due to the rarity of the disease, the number of patients in the study was relatively low. Use of a lower temporal resolution of the TRCE MR imaging in the initial phase of the study may have introduced some heterogeneity, though we believe that its effect on diagnostic accuracy is negligible.

\section{CONCLUSIONS}

VHHT2WI is superior in identifying SCAVMs, while the TRCE MR imaging is superior in identifying PMAVFs, with both sequences having equal accuracy in diagnosing SDAVFs. For feeder identification, VHHT2WI is superior in SDAVFs, while the other sequence is more useful in SCAVMs sand PMAVFs. The combined evaluation of VHHT2WI and TRCE MR imaging increases the sensitivity and specificity in identifying and classifying spinal vascular malformations and thus could be used for planning targeted DSA and subsequent interventions.

Disclosures: Bejoy Thomas_UNRELATED: Grants/Grants Pending: Department of Science and Technology, Department of Biotechnology, GE Healthcare*; Patents (Planned, Pending or Issued): 2 Indian patent applications pending*; Stock/Stock Options: shareholder Wipro India. Chandrasekharan Kesavadas-UNRELATED: Employment: Sree Chitra Tirunal Institute for Medical Sciences and Technology. *Money paid to the institution.

\section{REFERENCES}

1. Aminoff MJ, Logue V. The prognosis of patients with spinal vascular malformations. Brain 1974;97:211-18 CrossRef Medline

2. Krings T, Geibsprasert S. Spinal dural arteriovenous fistulas. AJNR Am J Neuroradiol 2009;30:639-48 CrossRef Medline

3. Toossi S, Josephson SA, Hetts SW, et al. Utility of MRI in spinal arteriovenous fistula. Neurology 2012;79:25-30 CrossRef Medline

4. Chen J, Gailloud P. Safety of spinal angiography: complication rate analysis in 302 diagnostic angiograms. Neurology 2011;77:1235-40 CrossRef Medline

5. Barreras P, Heck D, Greenberg B, et al. Analysis of $\mathbf{3 0}$ spinal angiograms falsely reported as normal in 18 patients with subsequently documented spinal vascular malformations. AJNR Am J Neuroradiol 2017;38:1814-19 CrossRef Medline

6. Gailloud P. Isolated aortic origin of the intersegmental spinal branch. J Neurointerv Surg 2013;5:e19 CrossRef Medline

7. Mull M, Nijenhuis RJ, Backes WH, et al. Value and limitations of contrast-enhanced MR angiography in spinal arteriovenous malformations and dural arteriovenous fistulas. AJNR Am J Neuroradiol 2007;28:1249-58 CrossRef Medline

8. Luetmer PH, Lane JI, Gilbertson JR, et al. Preangiographic evaluation of spinal dural arteriovenous fistulas with elliptic centric contrast-enhanced MR angiography and effect on radiation dose and volume of iodinated contrast material. AJNR Am J Neuroradiol 2005;26:711-18 Medline

9. Ali S, Cashen TA, Carroll TJ, et al. Time-resolved spinal MR angiography: initial clinical experience in the evaluation of spinal arteriovenous shunts. AJNR Am J Neuroradiol 2007;28:1806-10 CrossRef Medline

10. Amarouche M, Hart JL, Siddiqui A, et al. Time-resolved contrastenhanced MR angiography of spinal vascular malformations. AJNR Am J Neuroradiol 2015;36:417-22 CrossRef Medline

11. Saindane AM, Boddu SR, Tong FC, et al. Contrast-enhanced timeresolved MRA for pre-angiographic evaluation of suspected spinal dural arterial venous fistulas. J Neurointerv Surg 2015;7:135-40 CrossRef Medline

12. Mathur S, Bharatha A, Huynh TJ, et al. Comparison of time-resolved and first-pass contrast-enhanced MR angiography in pretherapeutic evaluation of spinal dural arteriovenous fistulas. AJNR Am J Neuroradiol 2017;38:206-12 CrossRef Medline

13. Kannath SK, Alampath P, Enakshy Rajan J, et al. Utility of 3D SPACE T2-weighted volumetric sequence in the localization of spinal dural arteriovenous fistula. J Neurosurg Spine 2016;25:125-32 CrossRef Medline

14. El Mekabaty A, Pardo CA, Gailloud P. The yield of initial conventional MRI in 115 cases of angiographically confirmed spinal vascular malformations. J Neurol 2017;264:733-39 CrossRef Medline

15. Kralik SF, Murph D, Mehta P, et al. Diagnosis of spinal dural arteriovenous fistula using 3D T2-weighted imaging. Neuroradiology 2017;59:997-1002 CrossRef Medline

16. Kannath SK, Rajendran A, Thomas B, et al. Volumetric T2-weighted MRI improves the diagnostic accuracy of spinal vascular malformations: comparative analysis with a conventional MR study. J Neurointerv Surg 2019 Mar 6. [Epub ahead of print] CrossRef Medline

17. Kannath SK, Thomas B, Sankara Sarma P, et al. Impact of non-contrast enhanced volumetric MRI-based feeder localization in the treatment of spinal dural arteriovenous fistula. J Neurointerv Surg 2017;9:178-82 CrossRef Medline 